\title{
Session 2: Therapeutic Applications I
}

\author{
Wednesday 14th April 2010. Moderator: Mark C. Glassy
}

[10.00-10.30]

'Monoclonal antibodies in the clinic, in the pipeline and in our dreams'

Don Capra

Oklahoma Medical Research Foundation, USA

Abstract not provided.

[10.30-11.00]

'Summary analysis of the pre-clinical and clinical results of brain cancer patients treated with pritumumab'

Mark C. Glassy

Nascent Biologics Inc., San Diego, California, USA

Abstract not provided.

[11.30-12.00]

'Combination therapies to enhance efficacy of cancer treatment'

Zdenka L. Jonak

GlaxoSmithKline, King-of-Prussia, Pennsylvania, USA

Abstract not provided.

[12.00-12.20]

'The epitope space as defined by proteomic similarity'

Darja Kanduc

University of Bari, Bari, Italy

An epitope is defined as a specific restricted antigen region that is capable of eliciting an immune response and of combining with a specific site (or paratope) of immunoglobulins. The specificity of the epitope-paratope interaction is at the core of the immune response and represents the foundations of immunology. However, after more than one century from the Ehrlichian antibody theory, we remain ignorant of the molecular and mechanistic factors that shape epitope-paratope interactions, determine antibody-antigen binding, and dictate antigen immunogenicity. During the last decade, we offered a robust set of experimental data suggesting that low level of sequence similarity to the host proteome modulates the B cell epitope repertoire in the humoral immune response. In parallel, a structured meta-analysis of scientific literature further supported our low-similarity theory by documenting that a low level of sequence identity to the host proteome was a minimum common denominator unifying the heterogeneous assembly of epitopes experimentally validated, described and used all over the world. The low-similarity theory has important implications in science and medicine. Scientifically, proteomic similarity analyses give a solution to the self-nonself discrimination issue that still lies unsolved in immunology reports. Clinically, low-similarity peptides might have a strong impact on the rational development of effective peptide-based treatments in cancer, infection and autoimmunity. In addition and surely of no least importance, the low-similarity offers the possibility of hitting the target with highest specificity and lowest cross-reactivity. Effective, safe and theoretically infallible immuno-therapeutical tools appear at hand.

[12.20-12.40]

'Tribody ${ }^{\mathrm{TM}}$ : A platform for novel antibody-derived biopharmacuticals'

Nico Mertens

Biotecnol SA, Oeiras, Portugal

Tribodies are multifunctional recombinant antibody derivatives, which utilise the naturalin vivo heterodimerization of the heavy chain ( $\mathrm{Fd}$ fragment) and light chain (L) of a Fabfragment, to form a scaffold, 
upon which additional functions can be incorporated, suchas additional binders - e.g. scFv binding domains.Each chain can be extended preferably at the Cterminus with an additional $\mathrm{scFv}$ binder. The chains are co-produced is produced in mammalian cells, where the host-cell BiPchaperone drives the formation of the heavy chain-light chain heterodimer (Fd:L) - thisreaction does not appear to be inhibited by the chain extensions. This leads to a veryspecific heterodimerization, using molecules abundantly present in serum (nonimmunogenic)These heterodimers are secreted into the media, where $>90 \%$ of the product were ofthe correct $\mathrm{Fd}: \mathrm{L}$ heterodimeric form. These are stable, with each of the bindersretaining their specific affinities, with the bivalent tribody having higher affinity, andhigher activatation of T-cell proliferation and cytotoxicityin vivo.This design allows easy engineering of multispecificity in a single molecule. Tribodies can be efficiently produced in mammalian cells and in the yeast Pichiapastoris. The main product is always the heterodimer, with some contamination of L-chain and Lchain dimer (Bence-Jones molecules), but these are easily removed bycation exchange chromatography. From cells adapted to serum free medium, a simplecombination of chromatography steps can yield pure product in a reproducible way.Although each molecule differs in yield and column behaviour, a general purificationscheme could be developed. Tribodies developed as a bispecific antibody either targeting two different tumorantigens (trispecific) or one tumor antigen bivalently, while monovalent targetingeffector cell activators (e.g. CD3 on T-cells) were produced and demonstrated activity.Also Immunocytokines with IL2 could be produced and were able to target the cytokineto the target cells.Cross-linking the CD3 at the tumor cell resulted in T-cell proliferation as well as activeand specific killing of the tumor cells in a mouse lymphoma model. In this model, therewas no need for additional CD28 or IL-2 stimulation. In a mouse B-cell lymphoma model, the Tribody behaved superior as compared tosc $(\mathrm{Fv}) 2$ (BiTe format) or the IgG1. 3 injections of 200 pmole $(15 \mu \mathrm{g})$ was sufficient toobtain $100 \%$ cure. These mice did not relapse.Tribodies are excellent scaffolds for constructing trivalent, bivalent bispecific, trispecificantibody derivatives or antibody-drug conjugates.

[12.40-13.00]

'BiTE antibodies in clinical trials - POC and a novel platform'

Tobias Raum

Micromet AG, Munich, Germany

BiTE antibody technology is based on single chain bispecific antibody molecules, binding to CD3 on cytotoxic T cells and a surface antigen on a target cell, such as a cancer cell. This close cell contact leads to the efficient killing of the target cell. The first BiTE antibody blinatumomab directed against CD19 on NHL cancer cells has proven efficacy in a clinical phase I trial. At a dose of $60 \mathrm{ug}$ per square meter body surface per day administered for 4 weeks as continuous i.v. infusion the objective response rate was $100 \%$ in seven patients (2 CR, 5 PR). Preliminary results from 16 evaluable patients of a phase II clinical trial in an B-ALL setting with complete haematological remission but molecular relapse $(\mathrm{MRD}+)$ indicate that treatment with blinatumomab can lead to conversion of MRD positivity into a MRD negative status in $81 \%$ of the patients.

An advanced new BiTE platform was recently developed, which is designed to generate BiTE antibodies that are cross-reactive between human and monkey target antigens to allow for early risk reduction with the clinical candidates in monkey studies. Results of such monkey studies using new BiTE antibodies will be presented. 